\title{
Osmo deshidratación del aguaymanto (Physalis peruviana)
}

\author{
Osmotic dehydration of aguaymanto (Physalis peruviana) \\ Ángel Quispe Talla ${ }^{1}$ y Nelly Castro Vicente ${ }^{1}$
}

\section{RESUMEN}

La investigación evalúa la prolongación de la vida útil del aguaymanto con las medidas biométricas y sus caracterizaciones, así como su higienización con agua ozonificada mediante la osmo deshidratación, en el comportamiento del jarabe y la evaluación de la característica de calidad del aguaymanto osmo deshidratado, estandarizándose el pelado químico con $2 \%$ de soda y temperaturas de $80^{\circ} \mathrm{C}$ y $85^{\circ} \mathrm{C}$ con tiempos de inmersión de 2 minutos. Las pulpas de aguaymanto se sometieron a los jarabes y soluciones de azúcar y de miel de abeja de $35,45,50$ grados Brix, a temperaturas de $25^{\circ} \mathrm{C}, 30^{\circ} \mathrm{C}$ y $35^{\circ} \mathrm{C}$ con tiempos de inmersión de $150 \mathrm{~min}, 180 \mathrm{~min}$ y $210 \mathrm{~min}$, respectivamente; alcanzando así una humedad del 22\%. Los parámetros para la osmo deshidratación de soluciones de miel de abeja utiliza un $50 \%$ de sólidos solubles a $35^{\circ} \mathrm{C}$ que en el lapso de 150 minutos alcanza su equilibrio, y es posible prolongar la vida útil del aguaymanto por más de 30 días mediante el empleo de la osmo deshidratación directa sin alterar ni su forma ni su textura. La calidad sensorial indica su aceptabilidad y preferencia sobre los productos ofertados en el mercado.

Palabras clave: osmo deshidratación; agua ozonificada; sólidos solubles.

\footnotetext{
1 Universidad Nacional Santiago Antúnez de Mayolo. Huaraz, Perú.

(C) Los autores. Este artículo es publicado por la Revista Aporte Santiaguino de la Universidad Nacional Santiago Antúnez de Mayolo. Este es un artículo de acceso abierto, distribuido bajo los términos de la Licencia Creative Commons Atribución-NoComercial-CompartirIgual 4.0 Internacional. (http://creativecommons.org/licenses/ by-nc-sa/4.0/), que permite el uso no comercial, distribución y reproducción en cualquier medio, siempre que la obra original sea debidamente citada.
} 


\begin{abstract}
The research indicates the prolongation of the useful life of aguaymanto with the biometric measurements and their characterizations as well as their hygienization with ozonated water, by means of the osmo dehydration, in the behavior of the syrup and the evaluation of the quality characteristic of the dehydrated osmo aguaymanto that chemical peeling was standardized with 2 percent soda and temperatures of $80^{\circ} \mathrm{C}$ and $85^{\circ} \mathrm{C}$, with immersion times of 2 minutes. The aguaymanto pulps were subjected to syrups and solutions of sugar and honey of 35, 45, 50 degrees Brix, at temperatures of $25^{\circ} \mathrm{C}, 30^{\circ} \mathrm{C}, 35^{\circ} \mathrm{C}$ and immersion times: $150 \mathrm{~min}, 180 \mathrm{~min}, 210 \mathrm{~min}$, reaching a humidity of $22 \%$. The parameters for the osmo Dehydration with honey solutions are using $50 \%$ soluble solids at $52^{\circ} \mathrm{C}$, which, within 150 minutes, reaches its equilibrium, and it is possible to extend the useful life of aguaymanto for more than 30 days by employing the osmo direct dehydration without altering its shape or texture. The sensory quality indicates its acceptability and preference over the products offered in the market.
\end{abstract}

Keywords: osmo dehydration; ozonized water; soluble solids.

\title{
INTRODUCCIÓN
}

En la actualidad, existe una tendencia mundial por la investigación y desarrollo de técnicas de conservación de alimentos que permitan obtener productos de alta calidad nutricional, que sean muy similares en color, aroma y sabor a los alimentos frescos y que no contengan agentes químicos conservantes. Entre las técnicas que son objeto de investigación en frutas se halla la deshidratación osmótica. Esta técnica permite obtener productos que reúnen las características indicadas y además los costos de producción son más bajos, si se compara con las técnicas que emplean calor o frío para los diferentes procesos de deshidratación. Los fundamentos de esta técnica de la osmo deshidratación y la viabilidad de emplearla con el aguaymanto, el proceso que de manera general permite la aplicación con los factores que más influyen en la velocidad de deshidratación, las características y los usos de jarabes de alto valor y el empleo de estos como un atributo complementario a su valor funcional permiten determinar las cantidades y características del aguaymanto y de sus productos obtenidos.

Finalmente, se necesitan del desarrollo de paquetes tecnológicos que presenten resultados logrados con el uso del laboratorio especializado de frutas y hortalizas de la Facultad de Ingeniería de Industrias Alimentarias para el uso de sus líneas de procesamiento y la transferencia de tecnología a los empresarios y productores de aguaymanto, contribuyendo a los ejes ambientales, en el área de manejo y desarrollo sostenible de 
recursos naturales y al eje económico en el área de producción en la extracción y transformación de los recursos naturales.

Estas consideraciones han permitido que se tracen los objetivos siguientes:

- Prolongar la vida útil del aguaymanto mediante la osmo deshidratación.

- Estudio del comportamiento del jarabe en la deshidratación osmótica del aguaymanto.

- Evaluación de la característica de calidad del aguaymanto osmo deshidratado.

\section{MATERIALES Y MÉTODOS}

La investigación se realizó en los laboratorios especializados de la Facultad de Ingeniería de Industrias Alimentarias y en el laboratorio de Microbiología de Alimentos en la UNASAM.

Materia prima: Aguaymanto proveniente del Callejón de Huaylas.

Insumos: Agua ozonificada, agua tratada, soluciones de miel de abeja de 35, 45 y 50 grados Brix, empaque para frutas y medios de cultivos para análisis microbiológico.

Análisis: Humedad, sólidos solubles, pH (AOAC, 1999), textura, acidez titulable, recuento de mesófilos aerobios viables, coliformes fecales y Staphylococcus aureus.

Diseño de la investigación: Los pasos que se siguieron para el estudio se reportan en el diagrama:

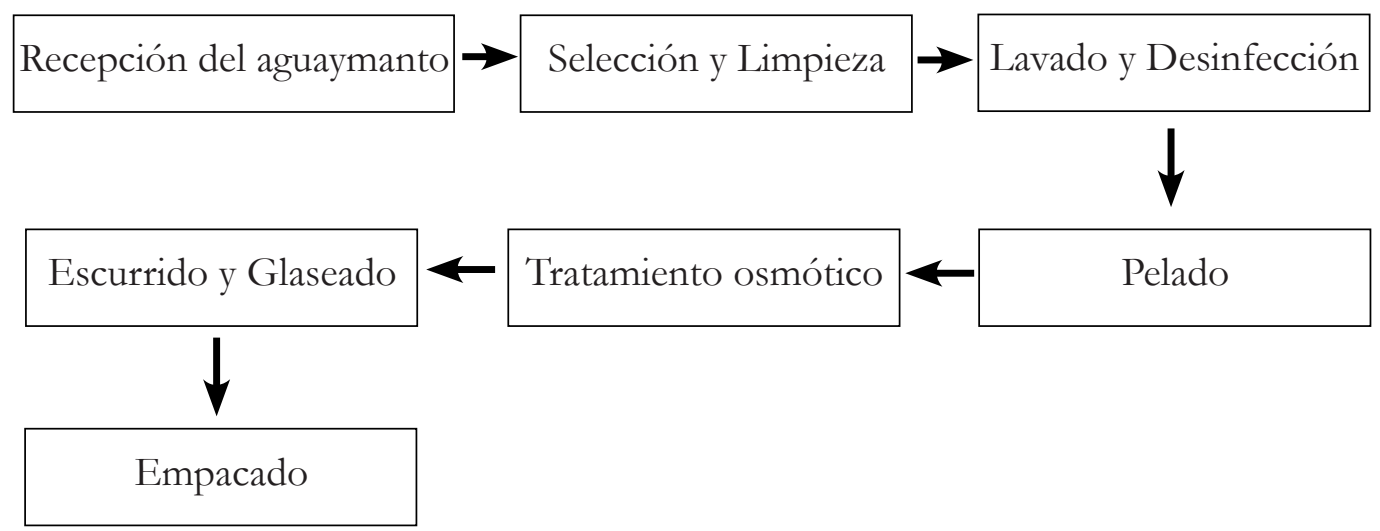

Figura 1. Diagrama de flujo para la osmosis deshidratación del aguaymanto 


\section{Caracterización de la materia prima}

a. Recepción: Los aguaymantos fueron recepcionados y colocados en un ambiente fresco y seco a temperatura de refrigeración $5-10^{\circ} \mathrm{C}$.

b. Selección y limpieza: Mediante inspección visual se eliminaron los productos deteriorados.

c. Higienización con agua ozonificada: Se higienizaron con agua ozonificada de 25 ppm de ozono.

d. Pelado de frutas: se pelaron químicamente con soda cáustica de 2 por ciento a $85^{\circ} \mathrm{C}$, se lavaron y neutralizaron con ácido cítrico a pH de 3,5 y se determinó su concentración de azúcar, humedad y peso.

e. Tratamiento osmótico: Se sumergieron en soluciones de miel de abeja de 35, 45, 50 grados Brix, a temperaturas de $25^{\circ} \mathrm{C}, 30^{\circ} \mathrm{C}$ y $35^{\circ} \mathrm{C}$ con tiempos de inmersión de 150 $\min , 180 \min$ y $210 \min$.

f. Empacado: El empacado se realizó en bolsas de policel y laminados, con sistema de atmósfera normal.

g. Evaluación sensorial: Se usó la prueba de aceptabilidad con panel seleccionado presentándole al juez dos muestras, de forma simultánea y se le solicitó que emita un juicio de aceptación eligiendo la muestra favorecida al analizar los atributos por separado como el dulzor y la textura, se les instruyó sobre las razones a tomarse en consideración para la calificación.

\section{RESULTADOS}

\section{Medidas biométricas}

Tabla 2. Medidas biométricas del aguaymanto promedio de 50 unidades

\begin{tabular}{ccc}
\hline Muestra & Diámetro $(\mathrm{cm})$ & Peso promedio $(\mathrm{g})$ \\
\hline Promedio & 2,033 & 4,25 \\
\hline
\end{tabular}



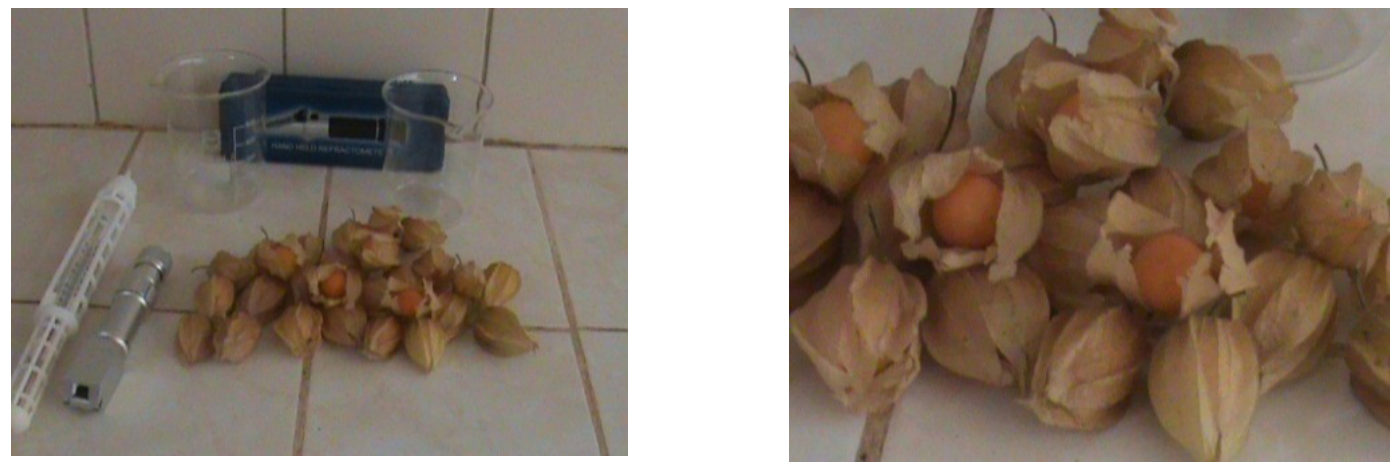

Figura 2. Aguaymanto antes de ser sometido a las medidas biométricas
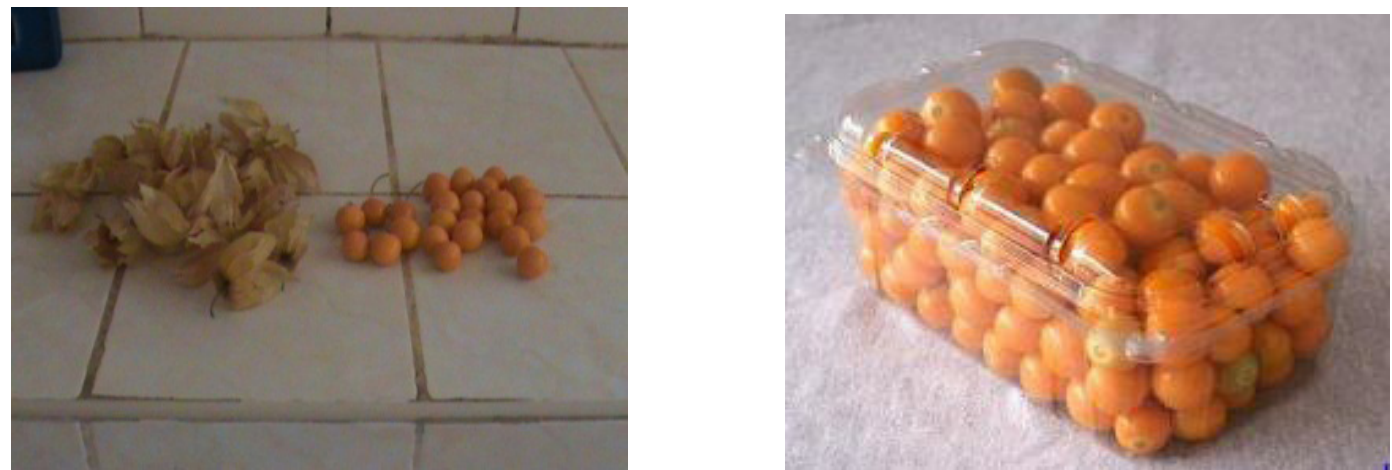

Figura 3. Aguaymanto para el reporte de sus medidas biométricas y almacenamiento para los tratamientos

\section{Grados Brix de la pulpa}

Tabla 3. Medidas de los grados Brix de la pulpa biométrica del aguaymanto promedio de 50 unidades

\begin{tabular}{ccc}
\hline Muestra & Sólidos solubles $\left({ }^{\circ}\right.$ Brix $)$ & Temperatura $\left({ }^{\circ} \mathrm{C}\right)$ \\
\hline Promedio & 14,0 & 20,0 \\
\hline
\end{tabular}



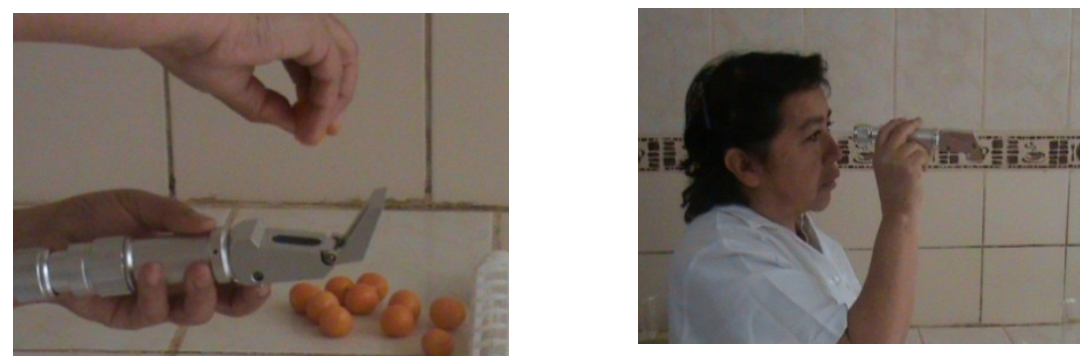

Figura 4. Determinación de los grados Brix del aguaymanto

Lavado y desinfección: Las frutas seleccionadas se higienizaron con agua ozonificada con 15 ppm y un tiempo de residencia de 8 minutos logrando la inocuidad del producto.

Pelado: Las frutas de aguaymanto se sometieron a un pelado químico en dos tratamientos por su estado de madurez que estas presentaron y después de su pelado fueron neutralizadas con soluciones de ácido cítrico en un $\mathrm{pH}$ de 4,0 y se determinó la actividad de la polifenol oxidasa.

Tabla 4. Tratamiento del pelado químico con soda cáustica del aguaymanto

\begin{tabular}{cccccc}
\hline Fruta & $\begin{array}{c}\text { Temperatura } \\
\left({ }^{\circ} \mathrm{C}\right)\end{array}$ & $\begin{array}{c}\text { Soda } \\
(\%)\end{array}$ & $\begin{array}{c}\text { Tiempo } \\
(\mathrm{min})\end{array}$ & $\begin{array}{c}\text { Actividad } \\
\text { Pelado }\end{array}$ & $\begin{array}{c}\text { Polifenoloxidasa } \\
\text { Aguaymanto }\end{array}$ \\
\hline Aguaymanto & 80 & 2,0 & 2,0 & Bueno & Ausente \\
\hline
\end{tabular}
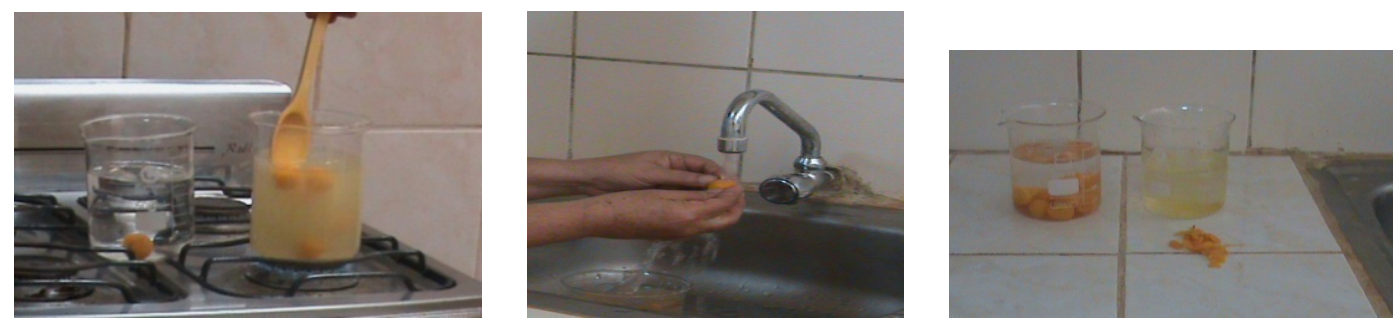

Figura 5. Pelado químico del aguaymanto

Tratamiento osmótico: Las pulpas peladas de aguaymanto se sometieron a los jarabes con soluciones de miel de abeja de 35,45 y 50 grados Brix, a temperaturas de $25^{\circ} \mathrm{C}$, $30^{\circ} \mathrm{C}$ y $35^{\circ} \mathrm{C}$ con tiempos de inmersión de $150 \mathrm{~min}, 180 \mathrm{~min}$ y $210 \mathrm{~min}$, respectiva- 
mente. A continuación se presenta las figuras de los sistemas de osmo deshidratación de las pulpas peladas de aguaymanto.
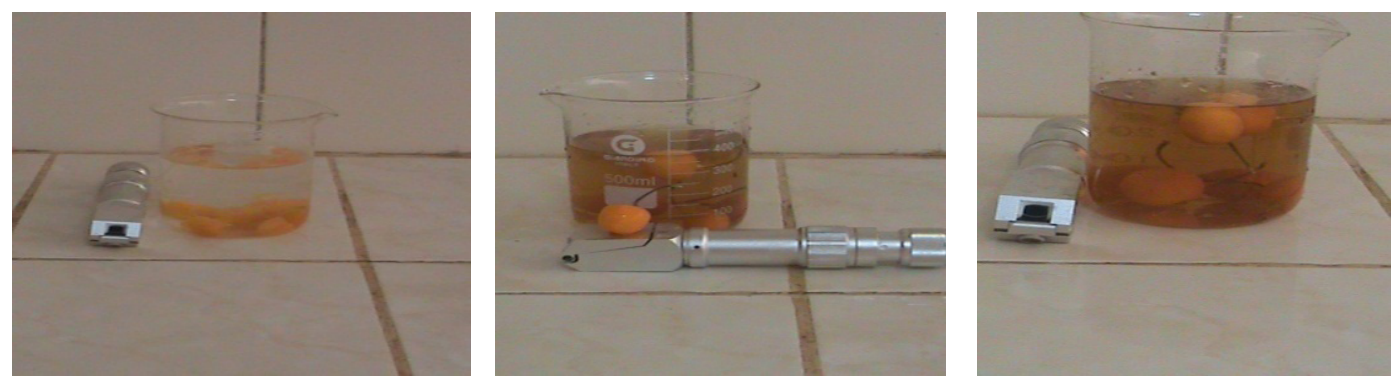

Figura 6. Sistemas de osmo deshidratación del aguaymanto peladas

Resultados de la osmo deshidratación: Los sólidos semi-infinitos se sumergieron en miel de abeja de 35, 45, y 50\% de sólidos solubles a fin de determinar la velocidad de deshidratación osmótica, evolución del peso del aguaymanto en la deshidratación osmótica directa, la variación de la concentración del jarabe durante el proceso osmótico, reducción porcentual de peso en función del tiempo en diferente concentraciones; para sólidos esféricos se muestran en los cuadros siguientes con sus respectivas gráficas.

Tabla 5. Pérdida de peso de los sólidos de forma esférica a $35^{\circ} \mathrm{C}$

\begin{tabular}{cccc}
\hline Tiempo (min) & $50 \%$ de SS & $45 \%$ de SS & $35 \%$ de \\
& $35^{\circ} \mathrm{C}$ & $35^{\circ} \mathrm{C}$ & $35^{\circ} \mathrm{C}$ \\
\hline 150 & 46,25 & 45,36 & 45,22 \\
105 & 44,75 & 44,26 & 43,67 \\
90 & 43,90 & 42,92 & 41,98 \\
75 & 42,10 & 41,60 & 39,58 \\
60 & 34,65 & 33,96 & 32,98 \\
45 & 25,32 & 22,60 & 20,32 \\
30 & 15,80 & 14,32 & 13,96 \\
15 & 10,20 & 9,250 & 9,01 \\
0 & 0,00 & 0,00 & 0,00 \\
\hline
\end{tabular}


Ángel Quispe Talla y Nelly Castro Vicente

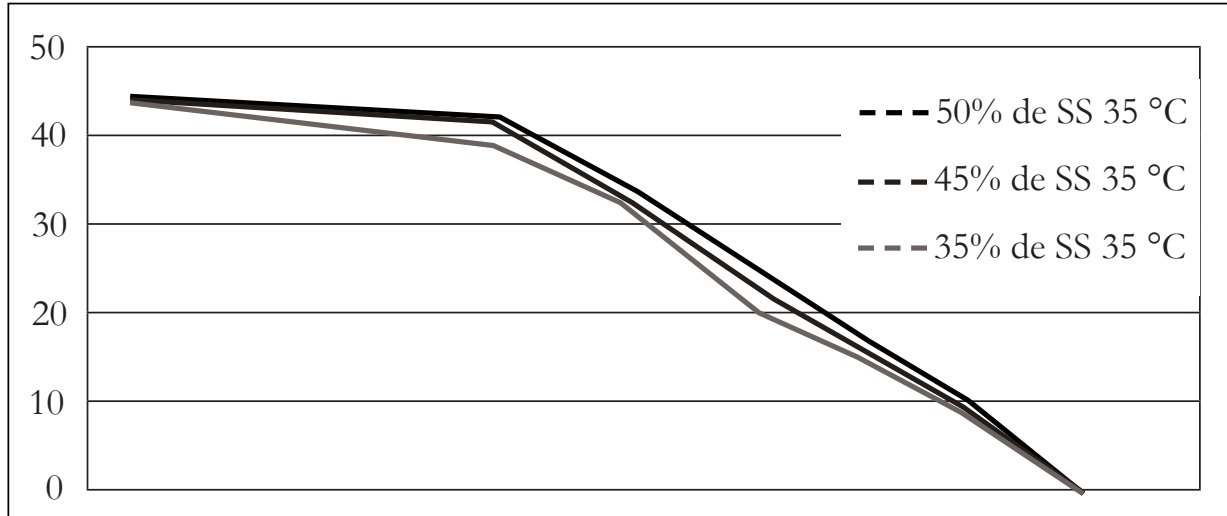

Figura 7. Comparación de la pérdida de peso de los sólidos esféricos a $35{ }^{\circ} \mathrm{C}$

Tabla 6. Pérdida de peso de los sólidos de forma esférica a $30^{\circ} \mathrm{C}$

\begin{tabular}{cccc}
\hline & $50 \%$ de SS & $45 \%$ de SS & $35 \%$ de \\
Tiempo (min) & $30{ }^{\circ} \mathrm{C}$ & $30^{\circ} \mathrm{C}$ & $30^{\circ} \mathrm{C}$ \\
\hline 180 & 46,13 & 45,38 & 45,20 \\
105 & 44,25 & 41,26 & 40,97 \\
90 & 39,25 & 36,88 & 38,65 \\
75 & 34,29 & 32,16 & 36,15 \\
60 & 30,76 & 24,85 & 29,16 \\
45 & 28,32 & 18,66 & 17,48 \\
30 & 22,10 & 19,62 & 15,73 \\
15 & 12,20 & 11,31 & 10,66 \\
0 & 0,00 & 0,00 & 0,00 \\
\hline
\end{tabular}

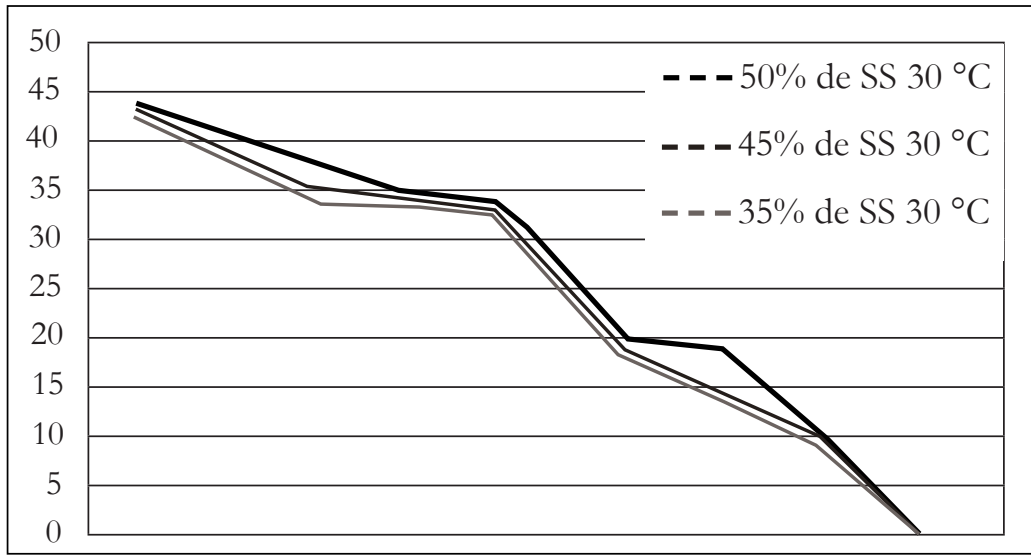

Figura 8. Comparación de la pérdida de peso de los sólidos esféricos a $30^{\circ} \mathrm{C}$ 
Tabla 7. Pérdida de peso de los sólidos de forma esférica a $25^{\circ} \mathrm{C}$

\begin{tabular}{cccc}
\hline Tiempo (min) & $50 \%$ de SS & $45 \%$ de SS & $35 \%$ de \\
& $25^{\circ} \mathrm{C}$ & $25^{\circ} \mathrm{C}$ & $25^{\circ} \mathrm{C}$ \\
\hline 210 & 44,25 & 43,36 & 42,22 \\
105 & 41,75 & 40,26 & 38,67 \\
90 & 38,90 & 35,88 & 34,35 \\
75 & 35,29 & 34,16 & 34,03 \\
60 & 33,76 & 32,85 & 31,79 \\
45 & 20,32 & 19,66 & 18,48 \\
30 & 18,710 & 15,92 & 14,93 \\
15 & 11,20 & 10,31 & 9,86 \\
0 & 0,00 & 0,00 & 0,00 \\
\hline
\end{tabular}

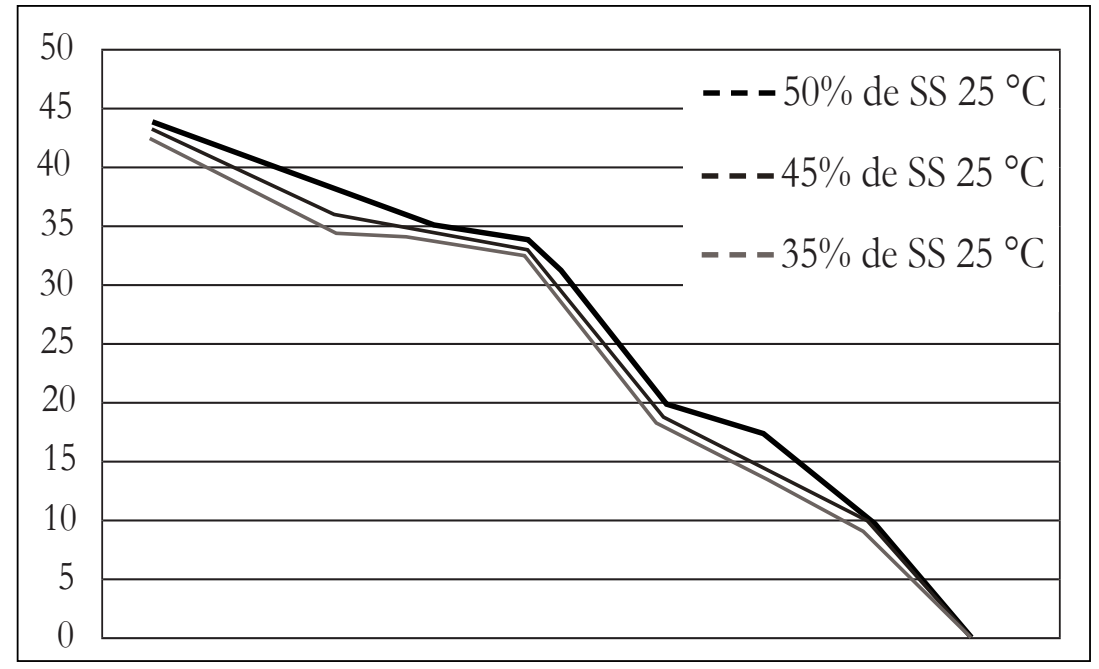

Figura 9. Comparación de la pérdida de peso de los sólidos esféricos a $30^{\circ} \mathrm{C}$

Escurrido y glaseado: El escurrido y glaseado se realizó con gelatina sin sabor a fin de darle una apariencia brillosa al osmo deshidratado con 15 gramos de gelatina base sin sabor por 200 gramos de agua presentándose el aguaymanto como si fuera marrasquinos de textura firme sin deformación de su geometría esférica, como se observa en la figura 10 . 

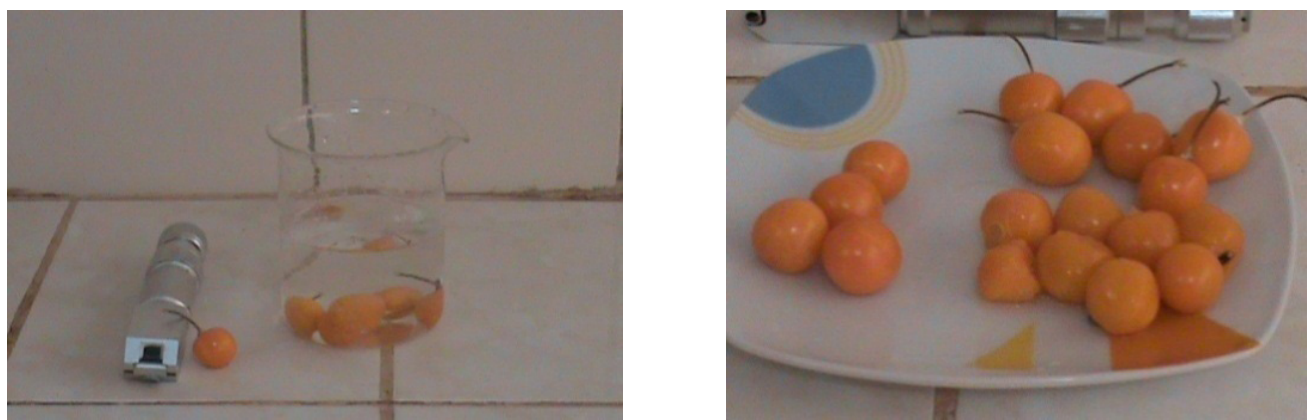

Figura 10. Proceso de glaseado y escurrido de pérdida de peso de los sólidos esféricos a $30^{\circ} \mathrm{C}$

Evaluación sensorial: Los resultados de la prueba de la evaluación sensorial se realizaron y se reporta en el cuadro siguiente.

Tabla 8. Resultado de la evaluación sensorial del aguaymanto osmo deshidratado en cubos

\begin{tabular}{|c|c|c|c|c|c|}
\hline \multirow[t]{2}{*}{ Panelista } & \multicolumn{4}{|c|}{ Scores para la muestra } & \multirow{2}{*}{$\begin{array}{c}\text { Panelistas } \\
\text { (X2) }\end{array}$} \\
\hline & A & B & $\mathrm{C}$ & $\mathrm{X}$ & \\
\hline 1 & 1 & 2 & 2 & 5 & 25 \\
\hline 2 & 2 & 3 & 4 & 9 & 81 \\
\hline 3 & 1 & 1 & 2 & 4 & 16 \\
\hline Totales & 4 & 6 & 8 & 18 & 122 \\
\hline
\end{tabular}

Tratamiento $\mathrm{X}=$ Totales separadamente para A, B y C (4, 6 y 8)

Panelista $\mathrm{X}=$ Totales separadamente para cada panelista $(5,9$ y 4$)$

Panelista $(\mathrm{X})^{2}=$ Cuadrado de los totales de arriba $(25,81$ y 16)

Elevando al cuadrado cada uno de los scores individualmente se obtiene el total (44)

Total X = sumando los cuatro scores (18)

Factor de corrección $(\mathrm{CF})=(\mathrm{X})^{2} / \mathrm{N}^{\mathrm{o}}$ de scores $=324 / 9=36$

Suma total de cuadrados del tratamiento $=\mathrm{X}^{2}-\mathrm{CF}$

$4^{2}+6^{2}+8^{2} / 3-36=116 / 3-36=2,67$

Suma de cuadrados del panelista $=122 / 3-36,0=4,67$ 
Tabla 9. Cómputo de F de la evaluación sensorial del aguaymanto osmo deshidratado

\begin{tabular}{cccccc}
\hline $\begin{array}{c}\text { Fuente de } \\
\text { variación }\end{array}$ & $\begin{array}{c}\text { Grados de } \\
\text { libertad }\end{array}$ & $\begin{array}{c}\text { Suma de } \\
\text { cuadrados }\end{array}$ & $\begin{array}{c}\text { Cuadrado } \\
\text { medio }\end{array}$ & F & $\begin{array}{c}\text { Nivel de } \\
\text { significancia }\end{array}$ \\
\hline Tratamiento & 2 & 2,67 & 1,33 & 8,06 & $5 \%$ \\
Panelista & 2 & 4,67 & 2,33 & 14,10 & $5 \%$ \\
Interacción & 4 & 0,66 & 0,165 & & - -.- $^{--}$ \\
Suma de & 8 & 8,00 & & & \\
variación & & & & & \\
\hline
\end{tabular}

Evaluación microbiológica: La tabla 10 presenta los resultados del análisis microbiológico donde se indica que es un alimento apto para consumo humano.

Tabla10. Análisis microbiológico del aguaymanto osmo deshidratado al inicio del almacenamiento y a los 30 días de almacenado

\begin{tabular}{cccc}
\hline Muestras de aguaymanto & Gérmenes viables & Hongos y levaduras & Coliformes \\
\hline Inicio del almacenamiento & Ausente & Ausente & Ausente \\
30 días de almacenamiento & Ausente & Ausente & Ausente \\
\hline
\end{tabular}

En las bolsas de polietileno de alta densidad no se manifestaron la presencia de cristales en el aguaymanto osmo deshidratado.

\section{DISCUSIÓN}

Las medidas biométricas que se reportan en los resultados de la tabla 2 y las figuras 3 y 4 concuerdan con el Ministerio de Agricultura (1990) y Jill (2004); se puede notar que presentan la forma esférica y pueden trabajarse en el proceso de osmo deshidratación como si fueran marrasquinos.

La tabla 3 y la figura 5 presentaron los resultados que correspondieron a los grados Brix de la pulpa del aguaymanto concuerdan con lo reportado con el Ministerio de Agricultura (1990) y Velezmoro (2004) presentando la pulpa a ese grado Brix una textura firme. Los grados Brix de la fruta permiten definir las concentraciones para los tratamientos del osmo deshidratado como lo indica Moyano (1991) y Lerici \& German (2005). 
Las frutas seleccionadas se higienizaron con agua ozonificada con 15 ppm y un tiempo de residencia de 8 minutos, como lo señala Castro (2010), lo que permitió obtener una fruta muy inocua, para la siguiente operación reportada en la tabla 3.

El pelado permitió determinar la esfericidad del sólido, esta disposición permite facilitar la transferencia de masa como lo indica Fernández (1992).

En el tratamiento osmótico, las pulpas peladas de aguaymanto se reportan en la figura 7 y estas alcanzaron su equilibrio en la transferencia de masa mediante la deshidratación osmótica a los 15 días de evaluación, como lo señala Lerici \& German (2005).

Respecto a la osmo deshidratación, la tabla 5 reporta a los sólidos semi - infinitos que se sumergieron en miel de abeja de 35, 45 y 50\% de sólidos solubles a fin de determinar la velocidad de deshidratación osmótica directa que originó la figura 8 , que presenta la gráfica de los sólidos esféricos de aguaymanto de 50\% sólidos solubles de miel de abeja, alcanza su máxima pérdida de peso a los 150 minutos en la osmo deshidratación directa, se observa que no pierde su forma y la difusión en la forma esférica permite una buena difusión como lo afirma Fernández (1992) con una humedad de 22\%.

La tabla 6 que origina la figura 9 se observa en el gráfico los sólidos esféricos de aguaymanto de 50\% sólidos solubles de miel de abeja, alcanza su máxima pérdida de peso a los 180 minutos en la osmo deshidratación directa, se observa que no pierde su forma y la difusión en la forma esférica, permite una buena difusión como lo afirma Fernández (1992) con una humedad del 24\%. La diferencia no es tan significativa con el tratamiento a $35^{\circ} \mathrm{C}$.

La tabla 7 origina la figura 11 donde se observa en la gráfica los sólidos esféricos de aguaymanto de 50\% sólidos solubles de miel de abeja, alcanza su máxima pérdida de peso a los 210 minutos en la osmo deshidratación directa, se observa que no pierde su forma, la difusión en la forma esférica es mejor, como lo afirma Fernández (1992) con una humedad del $23 \%$, y la diferencia no es tan significativa con el tratamiento a $35^{\circ} \mathrm{C}$ y $30^{\circ} \mathrm{C}$.

El escurrido y glaseado se realizó con gelatina sin sabor a fin de darle una apariencia brillosa al osmo deshidratado; como se indicó en la figura 11 mantiene su forma esférica sin deformación de su geometría con textura firme y dando la apariencia de marrasquinos.

En la tabla 8 se indica el análisis sensorial del panel seleccionado, que completado con la tabla 9 se aprecia que no existe una diferencia significativa debido a los panelistas así como la diferencia entre las muestras, por lo que se acepta el aguaymanto osmo deshidratado, las pruebas de evaluación sensorial fueron referidas como lo indica Ureña (1999). 
La tabla 10 presenta los resultados del análisis microbiológico donde se indica que es un alimento apto para el consumo humano; en las bolsas de polietileno de alta densidad no se manifestaron la presencia de cristales en el aguaymanto osmo deshidratado y la carga microbiológica estuvo dentro de los límites de control establecido por ITINTEC (1986).

\section{CONCLUSIONES}

La pulpa del aguaymanto tiene condiciones favorables para los procesos de osmo deshidratación por las medidas biométricas que presenta un diámetro de 2,033 cm y un peso de 4,25 gramos por fruto con un modelo espacial esférico con un radio de 1,0165 cm con $15^{\circ} \mathrm{C}$ brix.

La higienización de la fruta se realiza con agua ozonificada de 15 ppm y un tiempo de residencia de 8 minutos.

El pelado químico se realiza con soda de $2 \%$ de concentración a una temperatura de $80^{\circ} \mathrm{C}$ y un tiempo de contacto de 2 minutos permitiendo la inactivación de la Polifenoloxidasa.

Las pulpas peladas de aguaymanto se sometieron a los jarabes con soluciones de azúcar y en soluciones de miel de abeja de 35,45 y 50 grados Brix, a temperaturas de $25^{\circ} \mathrm{C}$, $30^{\circ} \mathrm{C}$ y $35^{\circ} \mathrm{C}$ y tiempos de inmersión de $150 \mathrm{~min}, 180 \mathrm{~min}$ y $210 \mathrm{~min}$, respectivamente, alcanzando una humedad final de $22 \%$.

Es posible prolongar la vida útil del aguaymanto por más de 30 días mediante el empleo de la osmo deshidratación directa obteniéndose un producto con $22 \%$ de humedad.

Los parámetros óptimos para la osmo deshidratación del aguaymanto en soluciones de miel de abeja es utilizando un $50 \%$ de sólidos solubles a $35^{\circ} \mathrm{C}$ que en lapso de 150 minutos alcanza su equilibrio.

Los resultados de la velocidad de osmo deshidratación indican que es más eficiente el modelo semi - infinito esférico logrando su estabilidad a los 150 minutos manteniendo su forma.

La evaluación sensorial realizada mostró que el producto es aceptable con un nivel de significación de $5 \%$.

Hasta los 30 días el producto osmo deshidratado no presenta carga microbiana que altere el producto teniendo este un contenido de humedad intermedia para su $22 \%$ de humedad. 


\section{REFERENCIAS BIBLIOGRÁFICAS}

AOAC. 1999. "Oficial Methods of Analysis”. Vol.-II 16ª Edición AOAC.

Castro, Nelly. 2010. Efecto del tratamiento al vacio en pulpas de manzana, durazno, mango y fresa minimamente procesadas en los laboratorios especializados de la FIIA. Huaraz: UNASAM.

Fernández, Carlos. 1992. "Determinación de la Difusividad efectiva de la sacarosa en la Papaya". Tesis. Lima: Universidad Nacional Agraria La Molina.

Giraldo, German. 2005. Deshidratación Osmótica como pre tratamiento de conservación de mora y Aguaymanto. Antioquia: Universidad de Antioquia.

ITINTEC. 1986. “Fruta confitada”. Norma Técnica 203.105. Lima.

Velezmoro, Jill. 2004. Perfil de mercado del Aguaymanto en Cajamarca. Cajamarca: Gobierno Regional de Cajamarca.

Lerici, Richard y German, F. 2005. "Osmotic Debydration of Fruit: Influence of Osmotic Agentson Drying Behavior and Product Quality”. Journal of Food Science. Vol. 50.

Ministerio de Agricultura. 1990. "Anuario de Piura y Tumbes”.

Moyano, Carmen. 1991. "Estudio Preliminar de la Difusión de Sorbato Durante la Deshidratación Osmótica de Manzana”. Actas del IV Congreso Latinoamericano de Transferencia de Calor y materia. 28-31 de octubre de 1991. Pp 356-358. Santiago: La Serna.

Ureña, Milber. 1999. Evaluación Sensorial de los alimentos. Lima: Universidad Nacional Agraria La Molina.

Recepción: 23/03/2017

Aceptación: 04/04/2018

\section{Correspondencia}

Ángel Quispe Talla

angelquispetalla@hotmail.com 